\title{
Correction to: GP-SLAM: laser-based SLAM approach based on regionalized Gaussian process map reconstruction
}

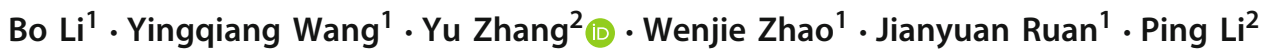

Published online: 7 March 2020

๑) Springer Science+Business Media, LLC, part of Springer Nature 2020

\section{Correction to:}

\section{Autonomous Robots}

https://doi.org/10.1007/s10514-020-09906-z

Unfortunately, the acknowledgement text was incorrectly published in the original article.

This has been corrected with this erratum.

Acknowledgements The paper is based upon work supported by the National Natural Science Foundation of China (Grant Nos. 61673341, 61703366, 61573091, 2016FZA4023), the Fundamental Research Funds for the Central Universities (Grant No. 2017QN81006), National Key R\&D Program of China (2016YFD0200701-3), the Project of State Key Laboratory of Industrial Control Technology, Zhejiang University, China (No. ICT1913), and the Open Research Project of the State Key Laboratory of Industrial Control Technology, Zhejiang University, China (No. ICT1900312).

Publisher's Note Springer Nature remains neutral with regard to jurisdictional claims in published maps and institutional affiliations.

The original article can be found online at https://doi.org/10.1007/ s10514-020-09906-Z.

Yu Zhang

zhangyu80@zju.edu.cn

Bo Li

jameslb20@hotmail.com

Yingqiang Wang

wangyinqiang@zju.edu.cn

Wenjie Zhao

zhaowenjie8@zju.edu.cn

Jianyuan Ruan

ruanjy@zju.edu.cn

Ping Li

pli@iipc.zju.edu.cn

1 School of Aeronautics and Astronautics, Zhejiang University, Hangzhou, China

2 State Key Laboratory of Industrial Control Technology, College of Control Science and Engineering, Zhejiang

University, Hangzhou, China 